\title{
Redatuming Operators Analysis in Homogeneous Media
}

\author{
Fransisco de Souza OLIVEIRA ${ }^{1,4}$, \\ Jose J.S. de FIGUEIREDO ${ }^{1,2}$, and Lucas FREITAS ${ }^{3}$ \\ ${ }^{1}$ Faculty of Geophysics, Federal University of Para, \\ Belem, Brazil; e-mail: frasol@ufpa.br \\ ${ }^{2}$ National Institute of Petroleum Geophysics (INCT-GP), \\ Salvador, Brazil; e-mail: jadsom@ufpa.br (corresponding author) \\ ${ }^{3}$ Geoprocessados, Virlemosa, Mexico; e-mail: lucas.batista.freitas@gmail.com \\ ${ }^{4}$ Faculty of Meteorology, Federal University of Para, Belem, Brazil
}

\begin{abstract}
A redatuming operation is used to simulate the acquisition of data in new levels, avoiding distortions produced by near-surface irregularities related to either geometric or material property heterogeneities. In this work, the application of the true-amplitude Kirchhoff redatuming (TAKR) operator on homogeneous media is compared with conventional Kirchhoff redatuming (KR) operator restricted to the zero-offset case. The TAKR and the KR operators are analytically and numerically compared in order to verify their impacts on the data at a new level. Analyses of amplitude and velocity sensitivity of the TAKR and KR were performed: one concerning the difference between the weight functions and the other related to the velocity variation. The comparisons between operators were performed using numerical examples. The feasibility of the KR and TAKR operators was demonstrated not only kinematically but also dynamically for their purposes. In other words, one preserves amplitude (KR), and the other corrects the amplitude (TAKR). In the end, we applied the operators to a GPR data set.
\end{abstract}

Key words: Kirchhoff redatuming, true-amplitude Kirchhoff redatuming, amplitude analysis, travel-time analysis. 


\section{INTRODUCTION}

Wavefield redatuming is an operation that transforms seismic data based on the assumption of a new measurement surface. In other words, given a data set acquired on an initial surface, it generates a simulated data set as if it were measured on another surface. Among its applications, we have near-surface corrections (Cox 1999), OBC processing (Jin et al. 2011), dual-sensor streamer de-ghosting (Klüver 2009), and multiple attenuation (Wiggins 1988).

The wave-equation based redatuming operators are the most accurate ones. Over the years several approaches have been proposed: based on the Kirchhoff integral (Berryhill 1984), using the phase-shift method (Margrave and Ferguson 1999) and based on the common-focus point (CFP) technology.

The redatuming operators are especially costly in the pre-stack domain in which most applications occur. Though the Kirchhoff method is rather straightforward and efficient, it is still expensive compared to static correction and requires an accurate interval velocity field above the datum. In addition, the Kirchhoff scheme is applied to common source and receiver gathers; in other to relocate sources and receivers, respectively. An analytical Kirchhoff-type redatuming operator, based on straight ray approximation (SRD) (Alkhalifah and Bagaini 2006) fills the gaps between static correction and wave-equation redatuming. It uses the assumption of local homogeneity, potentially useful for most media. The small size of the operator and its analytical expression provides cost-effectiveness and little sensitivity to velocity errors.

Toward a true-amplitude Kirchhoff-type operator, particular cases of the migration to zero-offset (MZO) operator proposed by Tygel et al. (1998) were analytically formulated for zero-offset configuration on homogeneous models and compiled into a true-amplitude Kirchhoff redatuming (TAKR) operator (Oliveira et al. 2009, Pila et al. 2014). This operator provides correct kinematic and dynamic redatumed traces. The reader should notice that the term true-amplitude is used here on a more strict sense, beyond amplitude relativity preservation. More specifically, the amplitude is not only preserved within a given event for different offsets. In this case, the amplitude has its geometrical spreading component adjusted to honor the new measurement surface.

For seismic data processing, the restriction to zero-offset configuration limits the applicability of a redatuming operator to event repositioning (e.g., in moving a stack from floating to final datum). However, as described by Liu et al. (2007), zero-offset redatuming plays a more important role in imaging GRP profiles. Moreover, an amplitude-friendly processing sequence is of great importance since amplitude analysis of GPR profiles has applications in shallow aquifers characterization (Bradford 1999), determination of subsurface contaminant (Schmalza and Lennartzb 2002) and soil water content variations (Cassidy 2007) in hydrological studies, and archaeological prospection (Khwanmuang and Udphuay 2012, Zhao et al. 2013). 
In this work, we analyze the amplitude behavior of two Kirchhoff-based redatuming operators through homogeneous media for the zero-offset case: the operator described by Berryhill (1984) and the true-amplitude operator proposed by Pila et al. (2014). We illustrate the feasibility of our analysis for a couple of numerical examples and for the application in a GPR data set from Siple Dome-Antarctica.

\section{THEORETICAL BACKGROUND}

In this section, we show our mathematical analysis for amplitude and travel-time attributes at redatuming operation. Our analysis relies on the stationary phase method to analytically solve the Kirchhoff redatuming in 2.5 D homogeneous media restricted to a zero-offset configuration.

\subsection{5 D Kirchhof-based redatuming}

Kirchhoff redatuming is based on the integral formulation of Kirchhoff migration (Schneider 1978). Like its migration counterpart, the redatumed wavefield $U_{o}$ is calculated by successive weighted summations of input wavefield $U_{i}$ along proper trajectories. More specifically,

$$
U_{o}\left(\xi_{o}, t_{o}\right)=\frac{1}{\sqrt{2 \pi}} \int_{Z_{i}} W d^{1 / 2} U_{i}\left(\xi_{i}, t_{o} \pm \tau\right) d \xi_{i}
$$

where $\xi_{i}$ and $\xi_{o}$ are the horizontal coordinates of the input and output datums $Z_{i}$ and $Z_{o}, d^{1 / 2}$ denotes the half-derivative of the input wavefield $U_{i}$, $\tau$ is the travel-time between the output location $\left(\xi_{o}, Z_{o}\left(\xi_{o}\right)\right)$ and input location $\left(\xi_{i}, Z_{i}\left(\xi_{i}\right)\right), W$ is a properly chosen weight. In frequency domain, $d^{1 / 2}$ corresponds to $i \omega$. Note that the \pm sign is chosen appropriately whether the output datum is above $(-)$ or below $(+)$ the input datum.

In this work, we analyse the amplitude behavior of two Kirchhoff-based redatuming operators through homogeneous media for the zero-offset case: the preserving operator described by Berryhill (1984) and the true-amplitude operator proposed by Pila et al. (2014).

These operators are kinematically identical. As expected, when redatuming from datum $Z_{i}$ to $Z_{o}$, through a homogeneous medium with velocity $v$, the travel-time $\tau$ is directly calculated from the distance between input and output locations $d\left(\xi_{i}, \xi_{o}\right)$, namely

$$
\left.d\left(\xi_{i}, \xi_{o}\right)=\sqrt{\left(\xi_{o}-\xi_{i}\right)^{2}+\left(Z_{o}\left(\xi_{o}\right)-Z_{i}\left(\xi_{i}\right)\right)^{2}}\right] .
$$


However, while the former aims to preserve relative amplitudes by direct application of the Kirchhoff integral, the latter aims to adjust the geometrical spreading by direct application of the MZO operator. These objectives are reached by using different stacking weights. The amplitudes are preserved using the weight $W_{p a}$ given by

$$
W_{p a}=\frac{\phi\left(\xi_{i}, \xi_{o}\right)}{\sqrt{v d}},
$$

where

$$
\phi\left(\xi_{i}, \xi_{o}\right)=d^{-1}\left[Z_{o}\left(\xi_{o}\right)-Z_{i}\left(\xi_{i}\right)-\left(\xi_{o}-\xi_{i}\right) Z_{i}^{\prime}\left(\xi_{i}\right)\right]
$$

accounts for the incidence correction. On the other hand, the true-amplitudes are achieved incorporating a geometrical spreading adjusting term

$$
\mathcal{G}\left(\xi_{i}, \xi_{o}, t_{o}, v\right)=\frac{2 d}{v t_{o}}
$$

into the summation weights, generating the true-amplitude weight $W_{t a}$ given by

$$
W_{t a}=(1 \pm \mathcal{G}) W_{p a}=\left(1 \pm \frac{2 d}{v t_{o}}\right) \frac{\phi\left(\xi_{i}, \xi_{o}\right)}{\sqrt{v d}},
$$

where $t_{0}$ is the output time and $d$ is the distance between input and output locations.

\subsection{Analytical analysis of velocity sensitiveness}

A first understanding of the impact of velocity errors on both operators can be obtained by analytical analysis of a simple case. In order to achieve that, we derive the stationary phase approximation (Bleistein 1986) of Eq. 1 in the case of horizontal plane input and output datums and a horizontal reflector in a constant velocity medium.

In the case of a reflector located at depth $D$ and with unitary reflectivity, the zero-offset input data set $U_{i}\left(\xi_{i}, t\right)$ is given by

$$
U_{i}\left(\xi_{i}, \omega\right)=\frac{1}{4 \pi D} e^{-i \omega 2 D / v},
$$

where $v$ is the medium velocity. Therefore, when using a redatuming velocity $\nu$, Eq. 1 can be replaced by

$$
\begin{aligned}
U_{o}\left(\xi_{o}, \omega\right) & =\frac{1}{\sqrt{2 \pi}} \int_{Z_{i}} W\left(\xi_{i} ; \xi_{o}\right)\left(\frac{\sqrt{i \omega}}{4 \pi D} e^{-i \omega 2 D / v}\right) e^{ \pm i \omega 2 d\left(\xi_{i} ; \xi_{o}\right) / \nu} d \xi_{i} \\
& =\frac{1}{\sqrt{2 \pi}} \int_{Z_{i}}\left(W\left(\xi_{i} ; \xi_{o}\right) \frac{\sqrt{i \omega}}{4 \pi D}\right) e^{-i \omega \phi\left(\xi_{i} ; \xi_{o}\right)} d \xi_{i},
\end{aligned}
$$


where $\phi\left(\xi_{i} ; \xi_{o}\right)=2 D / v \pm 2 d\left(\xi_{i} ; \xi_{o}\right) / \nu$. For this simple case, it is easy to note that the phase $\phi\left(\xi_{i} ; \xi_{o}\right)$ is stationary when $\xi_{i}=\xi_{o}$. The redatumed sample $U_{o}\left(\xi_{o}, \omega\right)$ can then be approximated by

$$
\begin{aligned}
U_{o}\left(\xi_{o}, \omega\right) & \approx \sqrt{\frac{1}{i \omega \phi^{\prime \prime}\left(\xi_{o} ; \xi_{o}\right)}}\left(W\left(\xi_{o} ; \xi_{o}\right) \frac{\sqrt{i \omega}}{4 \pi D}\right) e^{-i \omega \phi\left(\xi_{o} ; \xi_{o}\right)} \\
& \approx \sqrt{\frac{\nu z}{2 i \omega}}\left(W\left(\xi_{o} ; \xi_{o}\right) \frac{\sqrt{i \omega}}{4 \pi D}\right) e^{-i \omega 2(D / v \pm z / \nu)} \\
& \approx W\left(\xi_{o} ; \xi_{o}\right)\left(\frac{\sqrt{\nu z}}{4 \pi D \sqrt{2}}\right) e^{-i \omega 2(D / v \pm z / \nu)}
\end{aligned}
$$

In the case of preserving amplitudes we use $W=W_{p a}$ (Eq. 5), after which we have

$$
\begin{aligned}
U_{o}\left(\xi_{o}, \omega\right) & \approx W_{p a}\left(\xi_{o} ; \xi_{o}\right) \frac{\sqrt{\nu z}}{4 \pi D \sqrt{2}} e^{-i \omega 2(D / v \pm z / \nu)} \\
& \approx\left(\frac{\sqrt{2}}{\sqrt{\nu z}}\right) \frac{\sqrt{\nu z}}{4 \pi D \sqrt{2}} e^{-i \omega 2(D / v \pm z / \nu)} \\
& \approx \frac{1}{4 \pi D} e^{-i \omega 2(D / v \pm z / \nu)}
\end{aligned}
$$

As expected, the redatumed signal has the same amplitude as the input data (Eq. 7), it does not depend on the chosen redatuming velocity $\nu$, and its phase is shifted by $z / \nu$.

In the case of taking into account the geometrical spreading factor we use $W=W_{t a}$ into the Fourier inverse of Eq. 9:

$$
\begin{aligned}
U_{o}\left(\xi_{o}, t_{o}\right) & \approx W_{t a}\left(\xi_{o} ; \xi_{o}\right) \frac{\sqrt{\nu z}}{4 \pi D \sqrt{2}} \delta\left(t_{o}-2(D / v \pm z / \nu)\right) \\
& \approx\left[\left(1 \pm \frac{2 z}{\nu t_{o}}\right) \frac{\sqrt{2}}{\sqrt{\nu z}}\right] \frac{\sqrt{\nu z}}{4 \pi D \sqrt{2}} \delta\left(t_{o}-2(D / v \pm z / \nu)\right) \\
& \approx\left(1 \pm \frac{2 z}{\nu t_{o}}\right) \frac{1}{4 \pi D} \delta\left(t_{o}-2(D / v \pm z / \nu)\right) .
\end{aligned}
$$

Note that in order to keep consistency, the \pm operator, chosen according to the redatuming direction, has an opposite meaning in the last equation. For example, when redatuming upwards, the first occurrence is - and the other 
is + . Namely, at the redatumed reflector we have

$$
\begin{aligned}
U_{o}\left(\xi_{o}, t_{o}=2(D / v \pm z / \nu)\right) & \approx\left(1-\frac{z}{D \nu / v+z},\right) \frac{1}{4 \pi D} \\
& \approx\left(\frac{\nu}{v}\right) \frac{1}{4 \pi(D \nu / v+z)}, \\
& \approx \frac{1}{4 \pi(D+z v / \nu)} .
\end{aligned}
$$

The true-amplitude redatuming operator, in order to properly account for geometrical spreading, relies on a proper choice of the redatuming velocity $\nu$. The redatumed amplitude is strongly dependent on the relation between the chosen redatuming velocity $\nu$ and the medium velocity $v$.

\section{RESULTS}

The validity of the analysis of amplitude and travel-time with a variation in the velocity model was confirmed by numerical and real data sets. In this section, we have performed our analysis on three homogeneous numerical examples and on a real GPR data set.

\subsection{Model I: two horizontal layers}

In this synthetic example, we apply redatuming operators in a horizontally layered model with two horizontal homogeneous layers, where the first acoustic wave velocity background was $v_{1}=2000 \mathrm{~m} / \mathrm{s}$ and the second was $v_{2}=$ $2200 \mathrm{~m} / \mathrm{s}$. The model that consists of two horizontal homogeneous layers is depicted in Fig. 1. The zero-offset data set (Fig. 2a), was simulated with a 25 $\mathrm{Hz}$ Ricker wavelet by Kirchhoff modeling and sampled at 4 ms every $10 \mathrm{~m}$. Its measurement level is constant at level $z=0 \mathrm{~m}$. Another data set, to be used as reference, was simulated with the measurement level equal to the target output

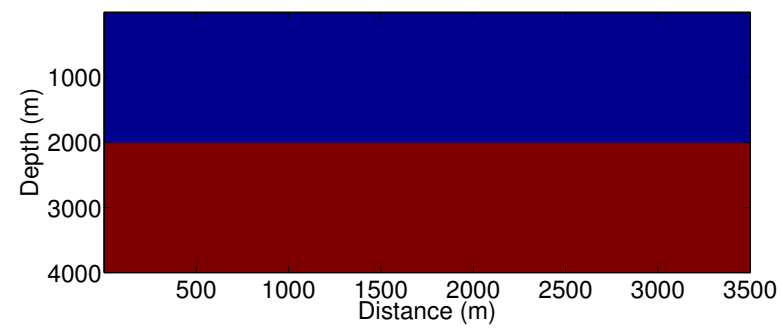

Fig. 1. Velocity model with two homogeneous layers (Model I). The first layer velocity is $2000 \mathrm{~m} / \mathrm{s}$ and the second $2200 \mathrm{~m} / \mathrm{s}$. 


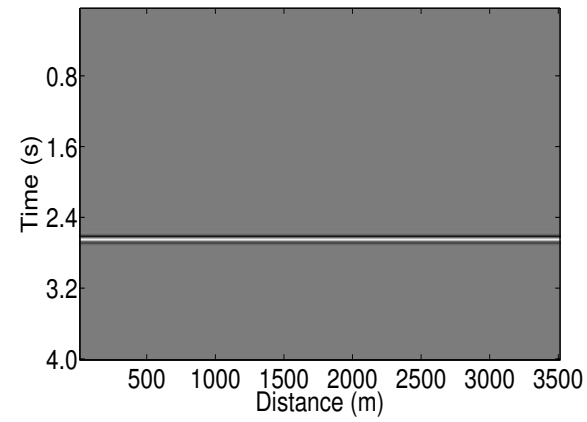

(a)

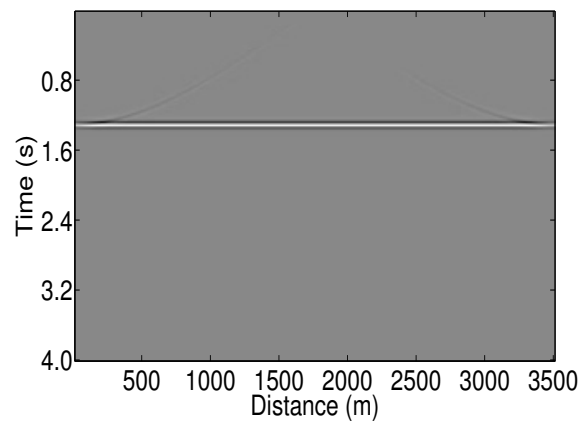

(c)

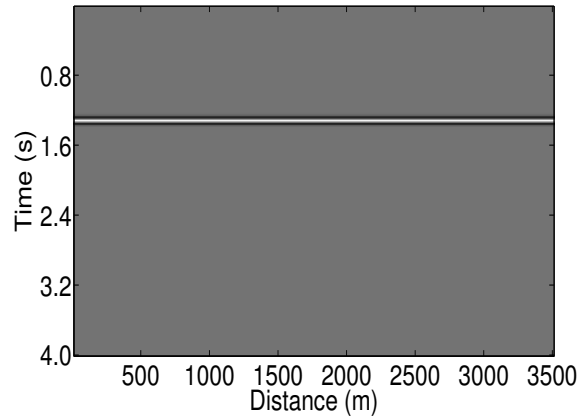

(b)

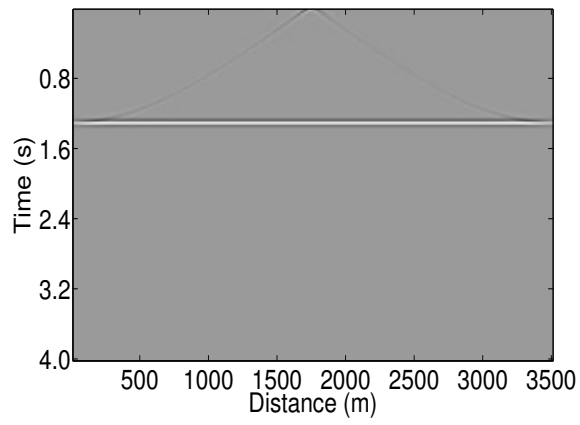

(d)

Fig. 2. Model I data sets: (a) input, (b) reference and outputs of (c) amplitudepreserving, and (d) true-amplitude redatuming operators.

datum ( $z=1000 \mathrm{~m}$ ) with identical parameters (see Fig. 2b). This datum level corresponds to $50 \%$ of the first layer depth.

The output of both true-amplitude and conventional redatuming operators is presented in Fig. 2c, d. A single-trace detailed comparison is presented in Fig. 3a. As expected, both operators result in traces kinematically equal to the reference trace. On the other hand, while the output from the conventional operator has the same amplitude as the input, the true-amplitude operator yields traces with the same amplitude as the reference trace. As we can notice in Fig. 3b, the amplitude erros are low and probably due to numerical issues.

A second experiment on the same model aimed to analyse the velocity sensitivity on both redatuming operators. Since the operators are kinematically the same, the errors in travel-time are identical (see Fig. 4a, b). On the other hand, as commented in Subsection 2.2, while the amplitude of the conventional operator is independent of the velocity error, the true-amplitude operator is considerably sensitive to it. The errors of the amplitude-preserving operator remain at $7 \%$ 


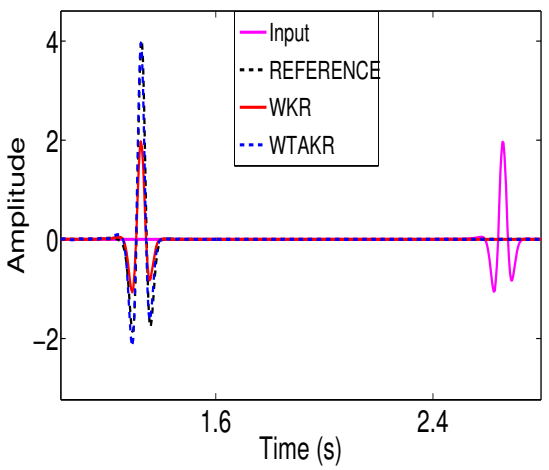

(a)

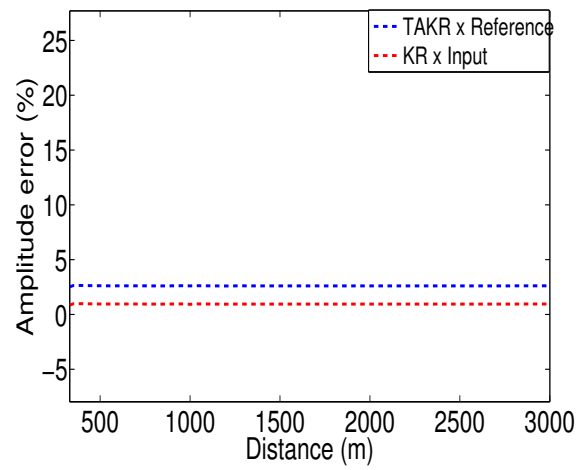

(b)

Fig. 3: (a) Seismic trace of the input (purple), reference (black dashed), KR (red), and TAKR (blue dashed). The KR and TAKR data were repositioned with the weight function (3) and (6), respectively, and the redatuming in this case recovered the correct amplitude; (b) The relative amplitude errors between the TAKR data and the reference (blue line) are around $3.5 \%$. The relative error between the KR and the reference data (red line) is around $1 \%$.

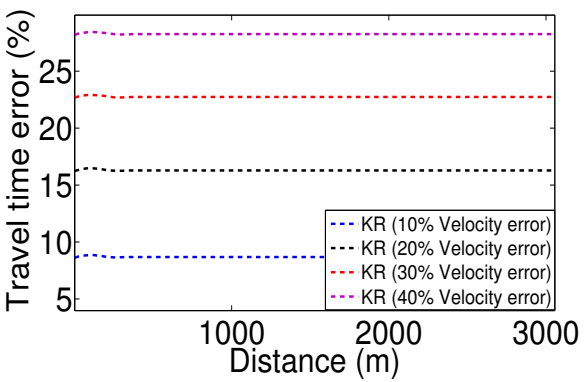

(a)

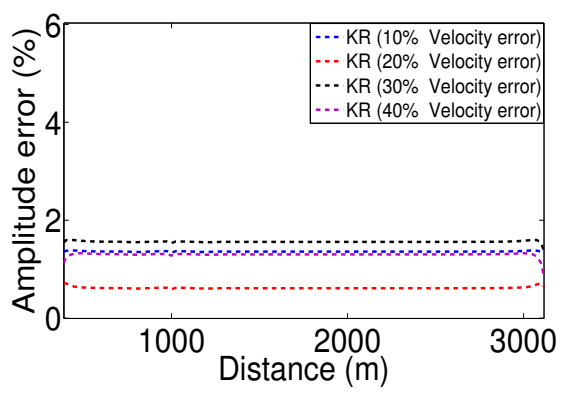

(c)

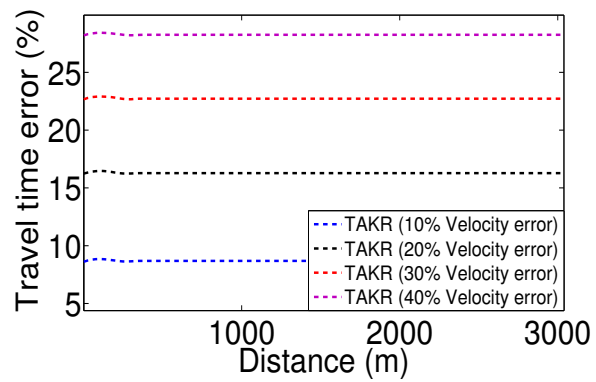

(b)

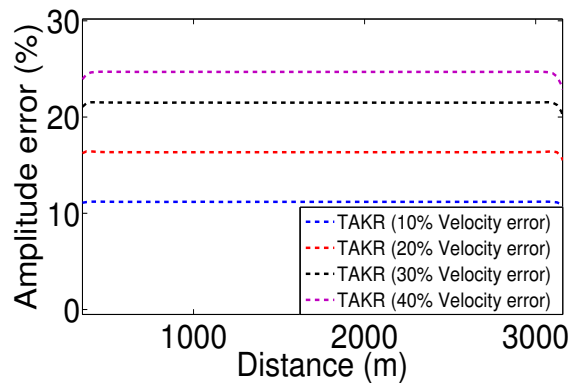

(d)

Fig. 4. Velocity error plots: traveltime error for (a) amplitude-preserving and (b) trueamplitude redatuming operators, and the respective amplitude errors shown in (c) and (d). 
even for velocity errors of $40 \%$, while the true-amplitude operator errors reach $27 \%$ in this case.

As it can be seen, both operators present accurate results, either preserving or adjusting the amplitude. However, in order to obtain an estimate of the geometrical spreading, the true-amplitude operator relies on a proper velocity model. Both analytic and numerical analyses show that the amplitude error for this operator is strongly affected by errors in the velocity field.

\subsection{Model II: four horizontal layers}

Here, in order to verify the feasibility of both operators when $V_{R M S}$ velocities are taken into account, we generated a second model which consists of four horizontal homogeneous layers, as depicted in Fig. 5. The interval velocities of this model were $1500,2000,2500$, and $3000 \mathrm{~m} / \mathrm{s}$.

The source-receiver pairs were positioned at every $25 \mathrm{~m}$. The data was modelled by Trisies (from Seismic Unix) which uses a Gaussian beam operator to produce true-amplitude synthetic seismograms with a Ricker wavelet of $25 \mathrm{~Hz}$. This seismogram was used as an input for KR operator in zero-offset. The zerooffset data set, shown in Fig. 6a has a sampling ratio of $4 \mathrm{~ms}$ and its measurement level is constant at $z=0 \mathrm{~m}$. Another data set, to be used as reference, was simulated having the measurement level equal to the target output datum ( $z=1000 \mathrm{~m}$ ) with identical parameters (see Fig. 6b) also by Trisies.

The outputs of both true-amplitude and conventional redatuming operators are presented in Fig. 6c, d. One-trace detailed comparisons are presented in Figs. 7 and 8. Just like the first experiment, both operators presented good accuracy in their kinematic and dynamic purposes.

As we can see Fig. 8, even when the $V_{R M S}$ velocity is used in the redatuming operation, the error between the KR and input data set is less than $1 \%$ for all layers, even deepest. However, in case of TAKR redatuming operation

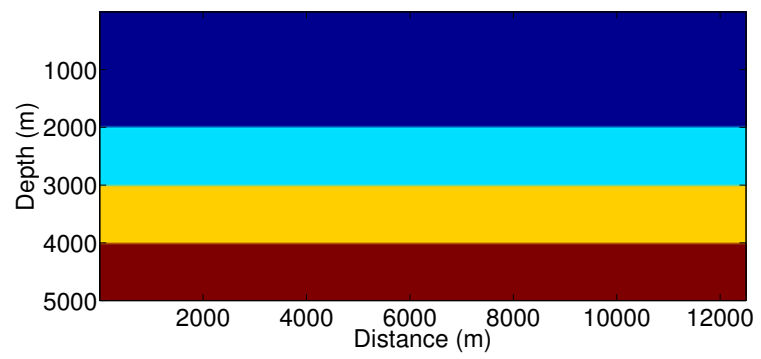

Fig. 5. Velocity model with four homogeneous layers with velocities 1500, 2000, 2500, and $3000 \mathrm{~m} / \mathrm{s}$ (Model II). 


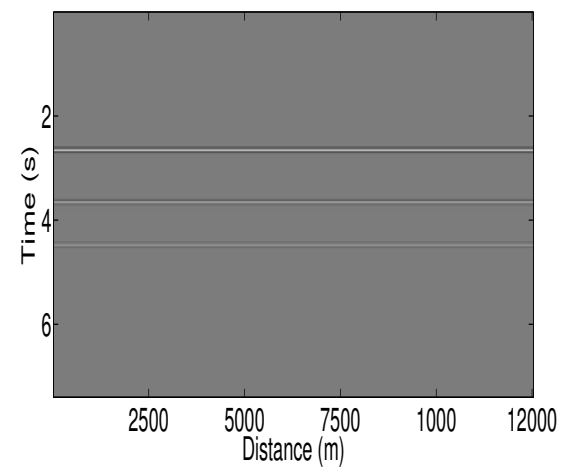

(a)

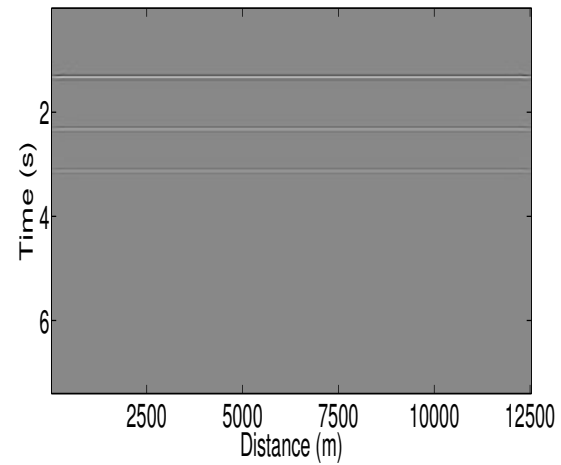

(c)

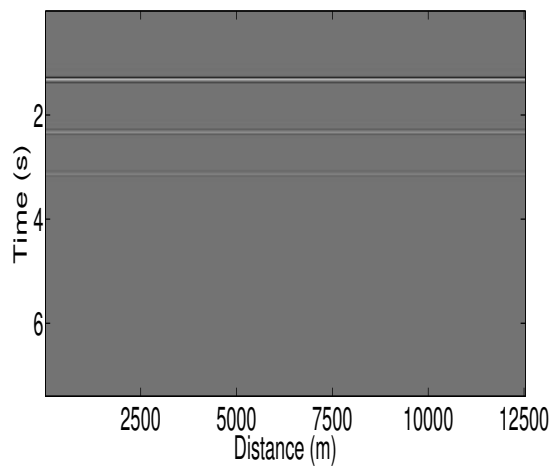

(b)

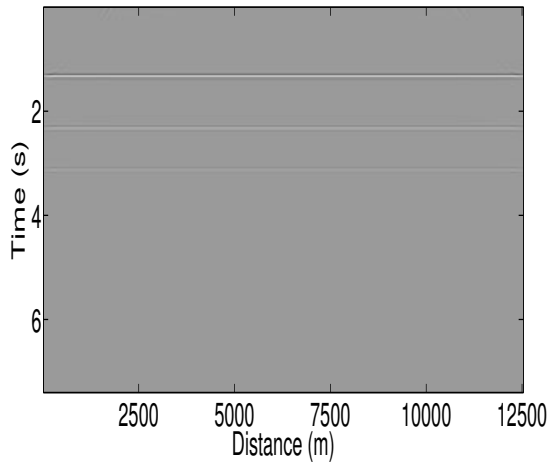

(d)

Fig. 6. Model II data sets: (a) input, (b) reference and outputs of (c) amplitudepreserving, and (d) true-amplitude redatuming operators.

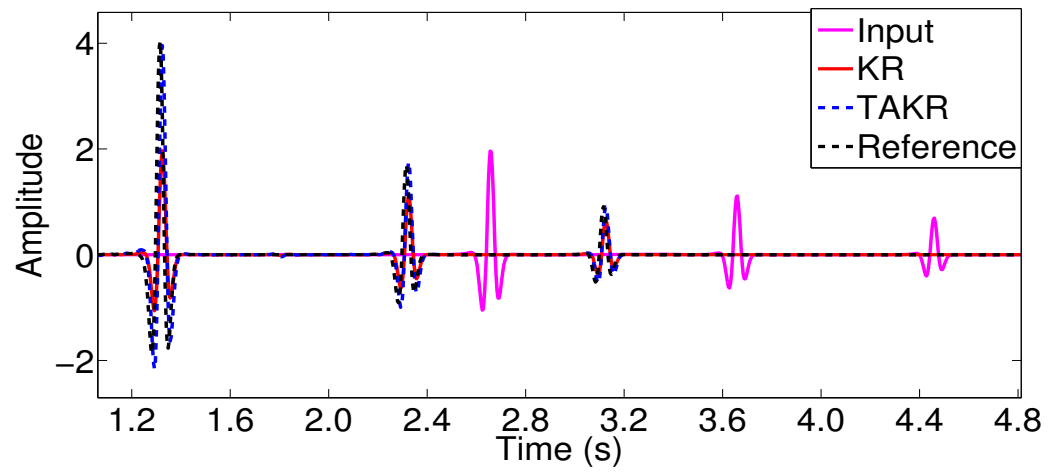

Fig. 7. Single-trace detailed comparison of Model II data sets. 


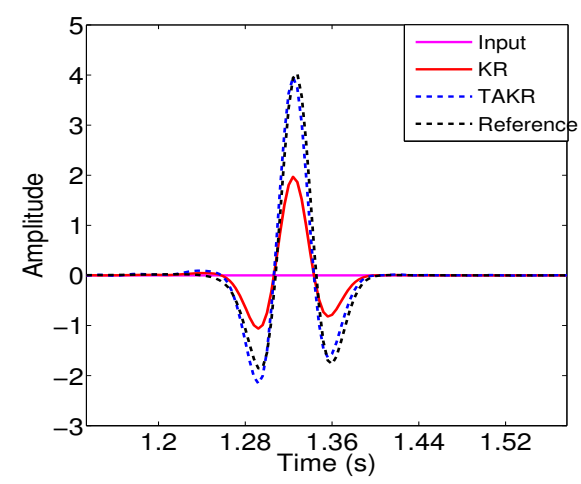

(a)

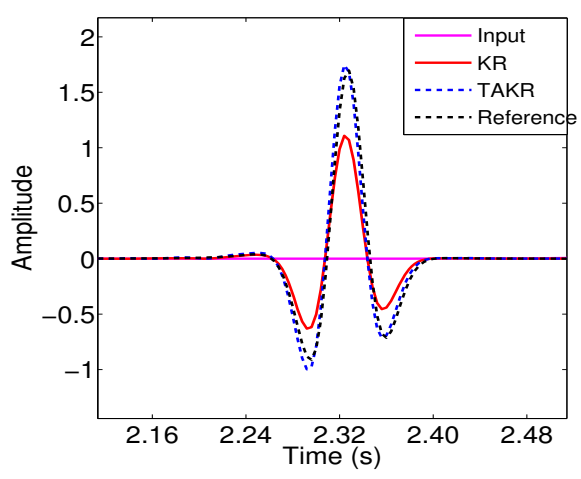

(c)

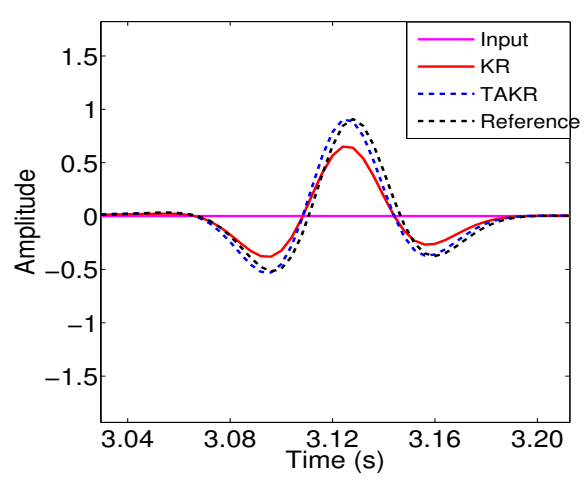

(e)

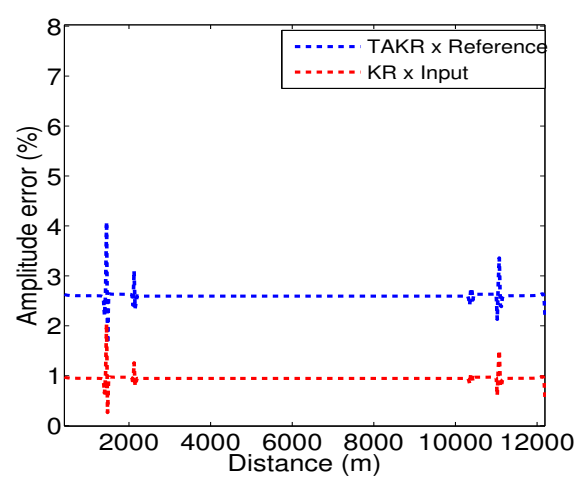

(b)

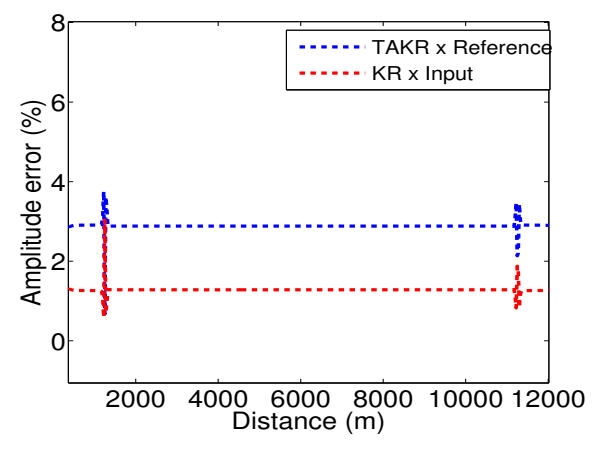

(d)

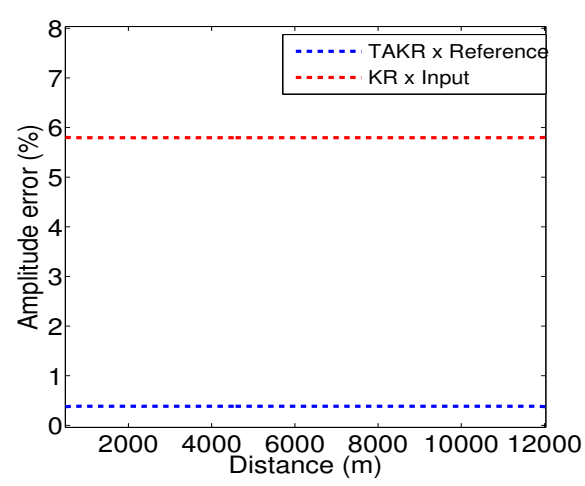

(f)

Fig. 8. Detailed comparison: (a), (c), and (e) single-trace zoom for first, second, and third events; (b), (d), and (f) multi-trace amplitude error plot for first, second, and third events. 
the error is more sensitive to differences between true and $V_{R M S}$ velocities. Based on what Subsection 2.2 shows, the TAKR is more sensitive to velocity error. The deeper the layer, the more $V_{R M S}$ moves away from interval velocity. It is expected the that TAKR operator is more sensitive to velocities of the deeper layers.

\subsection{Model III: four curved layers}

The third model consists of four curved homogeneous layers as depicted in Fig. 9. The zero-offset data set (Fig. 10a) was simulated with a $25 \mathrm{~Hz}$ Ricker wavelet by Gaussian beam modelling, sampled at $4 \mathrm{~ms}$ and $10 \mathrm{~m}$, and its measurement level is constant at $z=0 \mathrm{~m}$. Another data set, to be used as reference, was simulated having the measurement level equal to the target output datum $(z=200 \mathrm{~m}$ ) with identical parameters (see Fig. 10b).

The output of both true-amplitude and conventional redatuming operators are presented in Fig. 10c, d. One-trace detailed comparison is presented in Fig. 11.

Figure 11 compares the central trace of the KR and TAKR data with the central trace of the input and the reference sections. Just like in the model with four layers, this experiment showed that the difference in amplitude between the $\mathrm{KR}$ response and the input data is still the same with the increase of depth, and the TAKR response and reference data increased with the increase of depth. However, when it comes to amplitude recovering, the TAKR profile has the amplitude recovering factor.

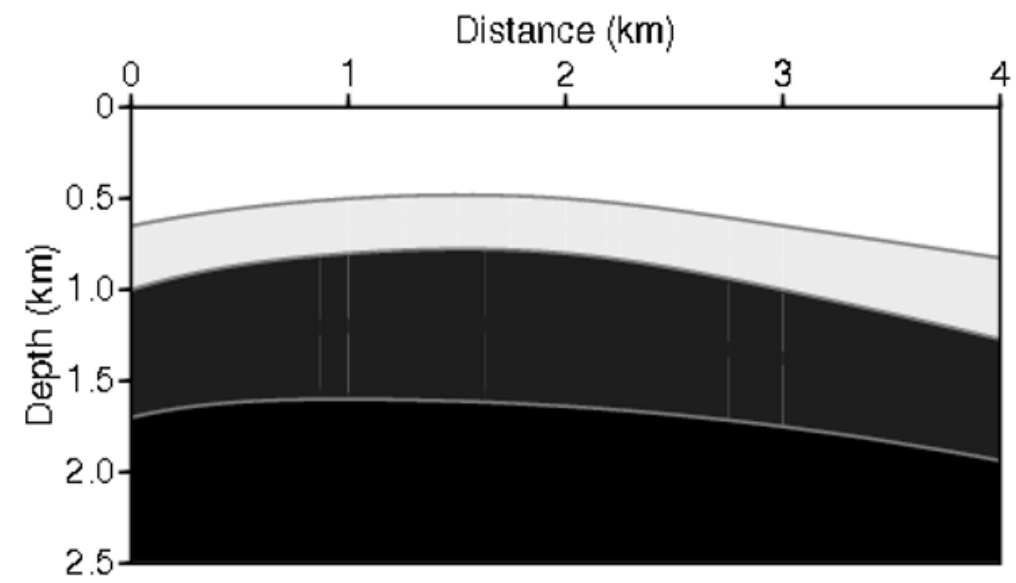

Fig. 9. Velocity model with four curved layers with velocities $1581,1690,1826$, and $2000 \mathrm{~m} / \mathrm{s}$ (Model III). 


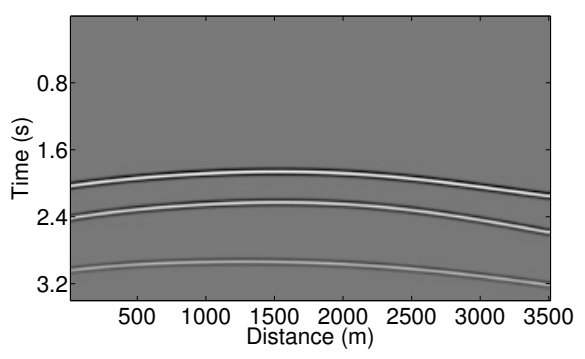

(a)

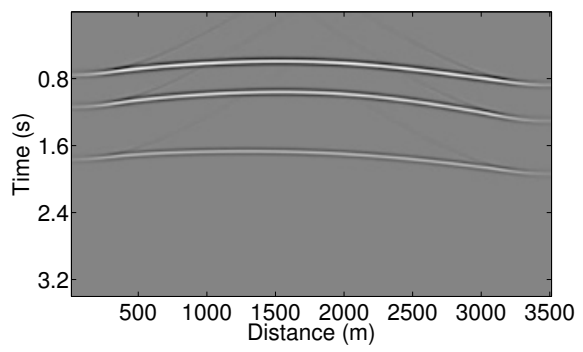

(c)

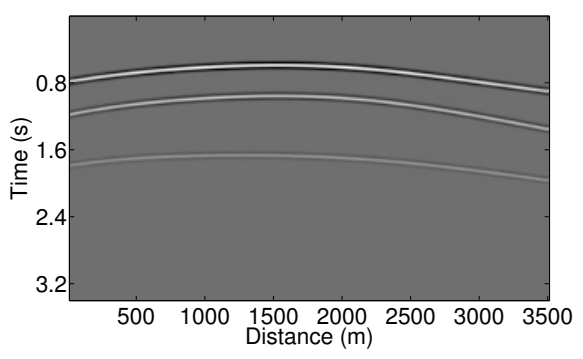

(b)

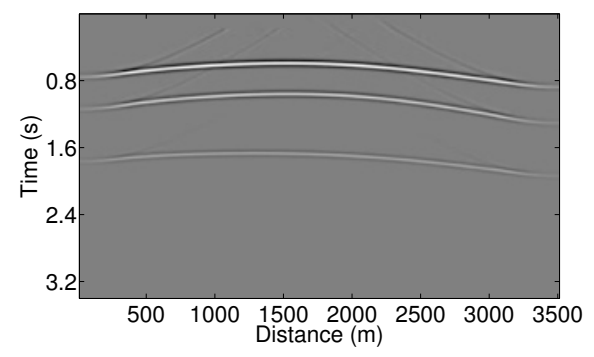

(d)

Fig. 10. Model III data sets: (a) input, (b) reference and outputs of (c) amplitudepreserving, and (d) true-amplitude redatuming operators.

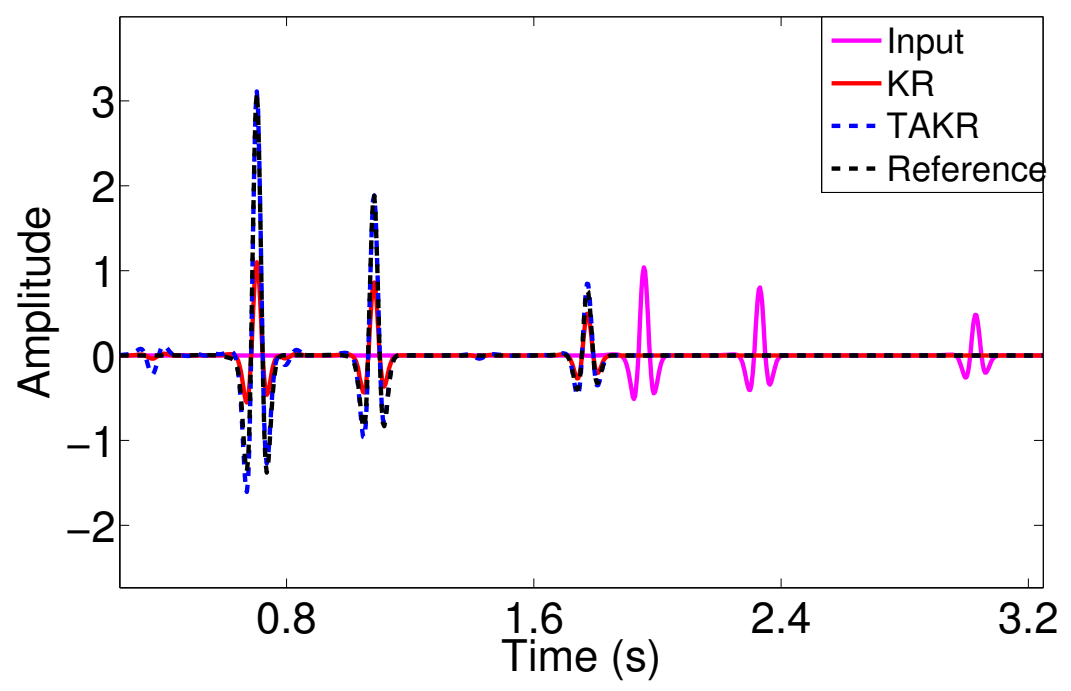

Fig. 11. Single-trace detailed comparison of Model III data sets. 


\section{FIELD DATA EXAMPLE:}

\section{GROUND PENETRATING RADAR (GPR) DATA SET}

Siple Dome (81.65 S, $148.81 \mathrm{~W}$ ) is an ice dome which is approximately $100 \mathrm{~km}$ wide and $100 \mathrm{~km}$ long, located $130 \mathrm{~km}$ east of Siple Coast in Antarctica. This place is of particularly important on determining the current mass balance of the West Antarctic ice sheet (WAIS).

The GPR survey was performed at a specific location indicated in Fig. 12a. Figure 12b shows a slice of the $100 \mathrm{MHz}$ GPR profile acquired in Siple Dome. The distance between the transmitter and receiver antennas was $1.0 \mathrm{~m}$ (halfoffset, $h=0.50 \mathrm{~m}$ ), and the interval between traces was $0.75 \mathrm{~m}$. A total of 8000 traces was collected along the $6000 \mathrm{~m}$ survey line. But here, for observation issues we are showing a spatial window of $3000 \mathrm{~m}$ (see Fig. 12b). The length of the time window was 913 ns and the number of samples per trace was 1870 , resulting in a time sampling rate of $0.49 \mathrm{~ns}$.

As an illustrative example, we applied both operators to the profile from Fig. 12b, redatuming it to a flat output datum located at $15 \mathrm{~m}$ depth. On a first example, we used ice velocity $(0.16 \mathrm{~m} / \mathrm{ns})$ as redatuming velocity (Alley and Bentley 1988). As expected, the difference between the two results is purely dynamic (see Fig. 13). While the conventional amplitude-preserving operator maintains the relativeness of the input profile (Fig. 13a), the true-amplitude operator boosts up amplitudes on the first $300 \mathrm{~ns}$ of the section. This enhancement

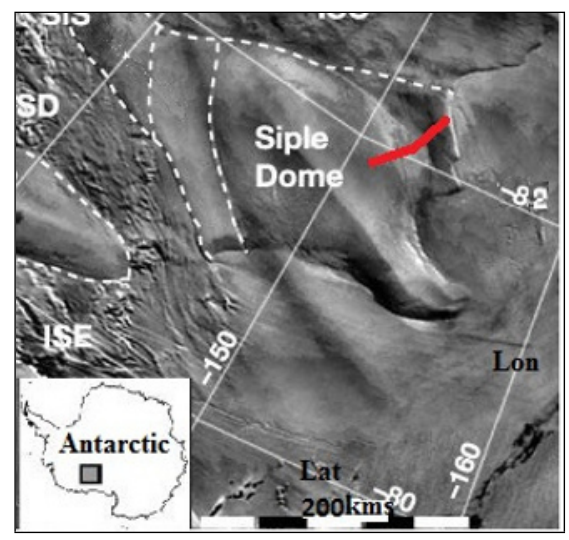

(a)

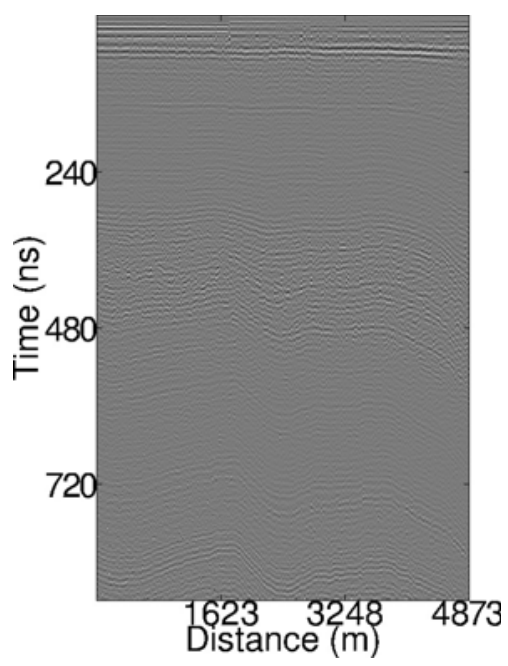

(b)

Fig. 12: (a) Map of location of Siple Dome in eastern Antarctica, Antarctic Explores; (b) Input GPR data $100 \mathrm{MHz}$ acquired in Siple Dome. 


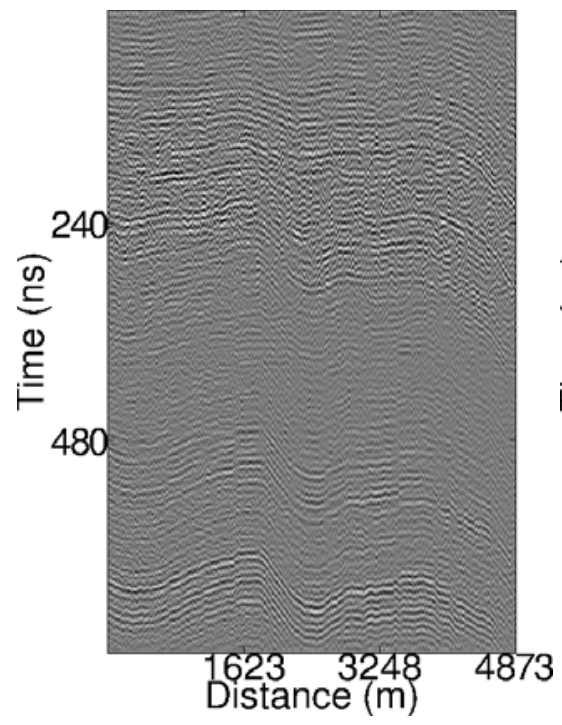

(a)

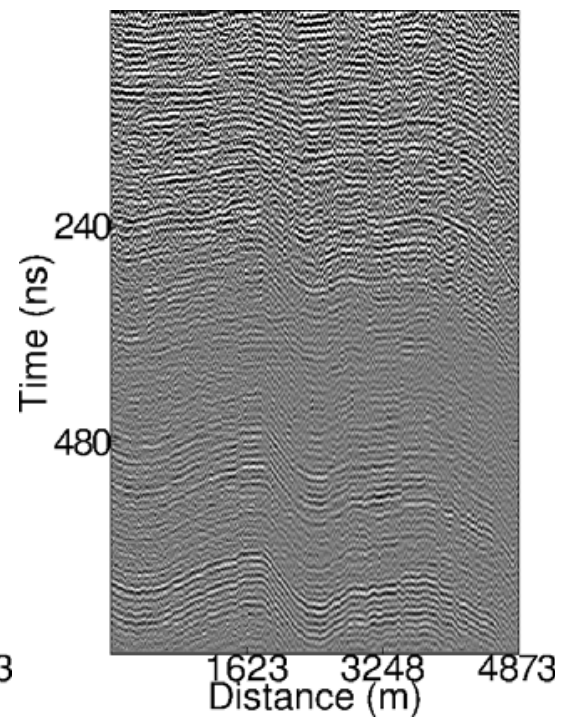

(b)

Fig. 13. Application of (a) amplitude-preserving, and (b) true-amplitude redatuming operator to profile of Fig. $12 b$.

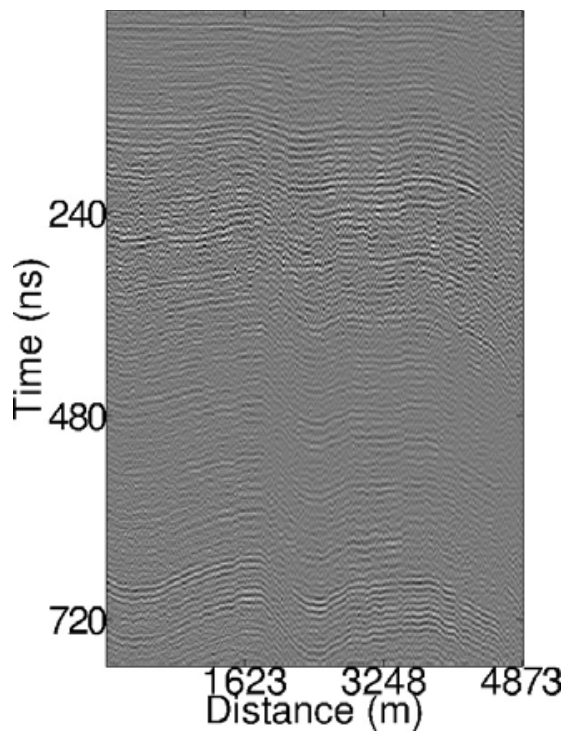

(c)

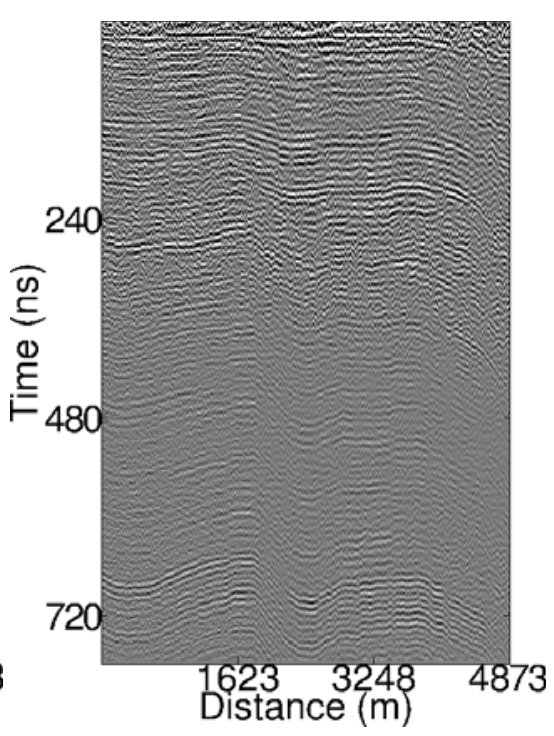

(d)

Fig. 14. Illustration of using an inacurate velocity field for (a) amplitude-preserving, and (b) true-amplitude redatuming. 
is more evident on the shallow part due to the bigger ratio between the acquired and adjusted geometrical spreading factors.

On a second example, in order to simulate the use of a non accurate velocity field, we chose a $50 \%$ higher redatuming velocity $(0.24 \mathrm{~m} / \mathrm{ns})$ when compared with ice velocity. As showed in the previous sections, Fig. 14 illustrates the impact of velocity error on both redatuming operators. Note that Figs. 13a and 14a present similar amplitude responses. On the other hand, the amplitude response of the true-amplitude operator is affected by the velocity error (see Figs. 13b and 14b).

As it can be seen, in both cases (with correct and wrong velocity) the amplitude at the deepest layers is highlighted for TAKR operation. In other words, the events presented better lateral continuity when compared with the TK result. We can observe these characteristics on the first events on the top of the data, on the left side around $300 \mathrm{~ns}$, and at the end in $700 \mathrm{~ns}$.

\section{CONCLUSIONS}

In this work, a comparative analysis was performed on two Kirchhoff redatuming operators: conventional and true-amplitude. When formulating both operators as variations of the same Kirchhoff integral operator, it become obvious that the difference between them is strictly dynamical and is due to a geometrical spreading correction factor at the stacking weight. This factor is responsible for replacing the input geometrical spreading by one adjusted to the new measurement surface. This difference is illustrated by numerical examples and one GPR field data set application. Using these examples, we illustrated that both operators fulfil their purposes, either preserving or adjusting the amplitudes.

Velocity sensitiveness analysies were also performed both analytically and numerically. We demonstrated that the true-amplitude operator is more sensitive to inaccuracies in the velocity field. While the conventional (amplitudepreserving) operator amplitude errors remain low, the true-amplitude operator amplitude errors increase (reaching $27 \%$ when in presence of $40 \%$ of velocity error).

The feasibility of our results were demonstrated through their application in GPR data acquired in a profile in Siple Dome-Antarctica. The TAKR and KR application to the GPR data showed that, in both cases, the reflectors were better delineated, presented better lateral continuity and the improved resolution of the main events, specially when the layers were deeper.

A c kn ow le d g me n t. The authors thank National Snow \& Ice Data Center for providing the GPR data. The authors appreciate the helpful comments and useful suggestions of Doctor Eric Verschuur. We thank Doctor Tiago A. 
Coimbra for his comments. This work was kindly supported by the Brazilian agencies CAPES, FINEP, and CNPq, as well as Petrobras.

\section{References}

Alkhalifah, T., and C. Bagaini (2006), Straight-rays redatuming: A fast and robust alternative to wave-equation-based datuming, Geophysics 71, 3, U37-U46, DOI: 10.1190/1.2196032.

Alley, R., and C. Bentley (1988), Ice-core analysis on the Siple Coast of West Antarctica, Ann. Glaciol. 11, 1-7.

Berryhill, J.R. (1984), Wave-equation datuming before stack, Geophysics 49, 11, 2064 2066, DOI: 10.1190/1.1441620.

Bleistein, N. (1986), Two-and-one-half dimensional in-plane wave propagation, Geophys. Prospect. 34, 5, 686-703, DOI: 10.1111/ j.1365-2478.1986.tb00488.x.

Bradford, J. (1999), Characterizing shallow aquifers with wave-propagation based geophysical methods: Imaging and attribute analysis, Ph.D. Thesis, Rice University, Houston, USA, http://hdl.handle.net/1911/18730.

Cassidy, N.J. (2007), Evaluating LNAPL contamination using GPR signal attenuation analysis and dielectric property measurements: Practical implications for hydrological studies, J. Contam. Hydrol. 94, 1-2, 49-75, DOI: 10.1016/j.jconhyd.2007.05.002.

Cox, M. (1999), Static Corrections for Seismic Reflection Surveys, Geophysical References, Vol. 9, Society of Exploration Geophysicists, Tulsa.

Jin, D., P. Yan, Q.-S. Tang, H.-B. Zheng, Y.-L. Wang, Q.-J. Lin, and J. Chen (2011), Application of Kirchhoff integral wave field extrapolation to water layer datuming for OBC record, J. Trop. Oceanogr. 30, 6, 84-89.

Khwanmuang, W., and S. Udphuay (2012), Ground-penetrating radar attribute analysis for visualization of subsurface archaeological structures, Leading Edge 31, 8, 946-949, DOI: 10.1190/tle31080946.1.

Klüver, T. (2009), Wavefield separation of dual-sensor towed streamer data using Kirchhoff type datuming and migration operators. In: 79th SEG Annual Meeting, 25-30 October 2009, Houston, USA, 2944-2948.

Liu, L., K. He, X. Xie, and J. Du (2007), Image enhancement with wave-equation redatuming: application to GPR data collected at public transportation sites, J. Geophys. Eng. 4, 2, 139-147, DOI: 10.1088/1742-2132/4/2/003.

Margrave, G.F., and R.J. Ferguson (1999), Wavefield extrapolation by nonstationary phase shift, Geophysics 64, 4, 1067-1078, DOI: 10.1190/1.1444614.

Oliveira, F., M. Pila, A. Novais, J. Costa, and J. Schleicher (2009), 2.5D True-amplitude diffraction-stack redatuming: numerical tests. In: Proc. 11th Int. Congr. of the Brazilian Geophysical Society "Seismic Processing: Migration", 1-6. 
Pila, M.F., J. Schleicher, A. Novais, and T.A. Coimbra (2014), True-amplitude singlestack redatuming, J. Appl. Geophys. 105, 95-111, DOI: 10.1016/j.jappgeo. 2014.03.010.

Schmalz, B., and B. Lennartz (2002), Analyses of soil water content variations and GPR attribute distributions, J. Hydrol. 267, 3-4, 217-226, DOI: 10.1016/S00221694(02)00152-X.

Schneider, W.A. (1978), Integral formulation for migration in two and three dimensions, Geophysics 43, 1, 49-76, DOI: 10.1190/1.1440828.

Tygel, M., J. Schleicher, P. Hubral, and L.T. Santos (1998), 2.5-D true amplitude Kirchhoff migration to zero offset in laterally inhomogeneous media, Geophysics 63 , 2, 557-573, DOI: 10.1190/1.1444356.

Wiggins, J.W. (1988), Attenuation of complex water-bottom multiples by waveequation-based prediction and subtraction, Geophysics 53, 12, 1527-1539, DOI: $10.1190 / 1.1442434$.

Zhao, W., E. Forte, M. Pipan, and G. Tian (2013), Ground penetrating radar (GPR) attribute analysis for archaeological prospection, J. Appl. Geophys. 97, 107117, DOI: 10.1016/j.jappgeo.2013.04.010.

Received 4 September 2013 Received in revised form 28 March 2014 Accepted 4 April 2014 